\title{
Region-Based Conditional Random Fields For Medical Image Labeling
}

\author{
YAN Yang ${ }^{1,2, a^{*}}$ \\ ${ }^{1}$ College of Communication Engineering, Jilin University, Changchun 130012, China; \\ ${ }^{2}$ College of Computer Science and Engineering, Changchun Normal University, Changchun 130032, \\ China \\ ayan_yang10@mails.jlu.edu.cn
}

Keywords: conditional random fields (CRFs), image labeling, over-segmented, medical image

\begin{abstract}
Concerning the high time complexity of medical image labeling in graph model, we proposed a region-based CRF method for medical image labeling. This method first over segmented the image into small homogeneous regions by using over-segmented method, and then the graphical model was constructed with regions as nodes and connecting the neighboring nodes as edges. The corresponding definition of region-based CRF were proposed and implemented. The experimental results shows that better medical image labeling results are obtained by the region-based CRF model. At the same time, running time is largely reduced, efficiency is improved.
\end{abstract}

\section{Introduction}

Medical image segmentation is the basis of medical image processing and analysis, accurate segmenting and identifying lesions and surrounding tissue with complex features is a prerequisite for early non-invasive and non-invasive examination and quantitative analysis, as well as an important foundation for follow-up treatment. However, due to the complexity of medical images, automatic segmentation method still cannot meet the clinical requirements, which is a leading-edge research direction in image processing.

Relationships between objects in medical images are often more complex, the randomness, and fuzziness, incomplete and unreliable of objective things often leads to uncertainty, probabilistic methods combine probability and graphical models into model-based approach, which provide an important way to address these uncertainties. CRF (Conditional Random Field) is a probabilistic graphical models proposed by Lafferty ${ }^{[1]}$, which is initially applied to natural language processing. In recent years, it has been successfully used in image processing and pattern recognition ${ }^{[2-5]}$, such as image segmentation, image classification, object detection and recognition, etc.

For the image segmentation problem, many methods such as logistic regression or support vector machines regard image pixel distribution as independent and identically distributed, however, the features of adjacent pixels are interdependent in fact. CRF reduces the probability distribution assumptions to achieve simpler and more adapted to contain a rich, overlapping image features, showing an excellent performance in the application, and achieved desired results ${ }^{[2-5]}$.

As all probabilistic graphical models, CRF is facing problems in the application as well, in medical image labeling, the data to be processed is two-dimensional image, it has great amount of data, which make the convergence speed of parameter estimation slower, calculation scale and the running time will be multiplied growing.

Therefore, we propose a region-based CRF for medical image labeling; the region represents a set of pixels which have similar features and space adjacent. We make region act as the node of graph model to reduce scale. Experimental results show that the region-based CRF model achieves desired results and accelerates running speed greatly at the same time.

In this paper, a conditional random field (CRF) model is used for object recognition and segmentation in medical images. By using an over-segmentation algorithm, we propose a new region based CRF model. We train our model on annotated samples based our model and then test. We compare our results with recent published results. The result shows that our region based CRF model significantly outperforms the current state-of-the-art. 


\section{Theory of Conditional Random Fields}

CRF can be represented as an undirected graphG $=(\mathrm{V}, \mathrm{E})$, whereV represents all the nodes (random variables), Eindicates undirected connection between the nodes. $\mathrm{X}$ represents the observed random variables(features), Y represents the unobserved random variables (label)。 The definition of Conditional Random Fields, as proposed by Lafferty ${ }^{[1]}$ is described below:

Let $G=(V, E)$ be a graph such that $Y=\left(Y_{v}\right)_{v \in V}$, so that $Y$ is indexed by the vertices of $G$. Then $(\mathrm{X}, \mathrm{Y})$ is a conditional random field, when conditioned on $\mathrm{X}$, the random variables $\mathrm{Y}_{\mathrm{v}}$ obeying the Markov property with respect to the graph:

$$
p\left(Y_{v} \mid X, Y_{w}, w \neq v\right)=p\left(Y_{v} \mid X, Y_{w}, w \sim v\right)
$$

Wherew $\sim \mathrm{V}$ means that $\mathrm{W}$ and $\mathrm{v}$ are neighbors in $\mathrm{G}$.

A CRF is thus a random field globally conditioned on the observation vector $\mathrm{X}$, by the fundamental theorem of random fields ${ }^{[6]}$, the joint distribution over the label sequence $\mathrm{Y}$ given $\mathrm{X}$ has the form:

$$
p(y \mid x)=\frac{1}{\mathrm{z}} \exp \left(\sum_{i \in V} A_{i}\left(y_{i}, x\right)+\sum_{i \in V} \sum_{j \in N_{i}} I_{i j}\left(y_{i}, y_{j}, x\right)\right)
$$

Where Zis the normalizing constant known as the partition function, and $A_{i}$ and $I_{i j}$ are the negative unary (association) and pairwise (interaction) potentials respectively.Graphical structure of chain-structured CRFs for sequences can be represented by Figure1.

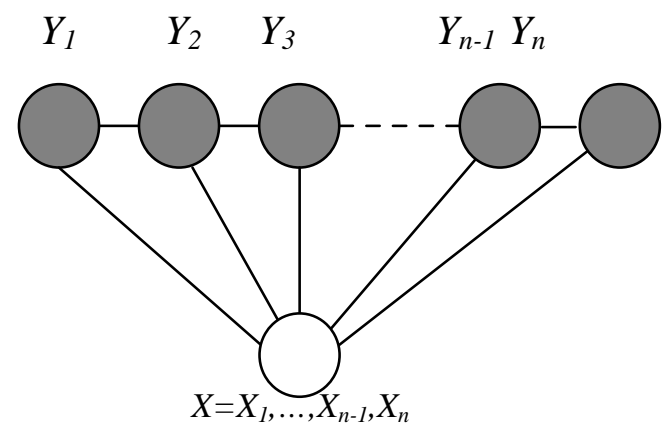

Fig.1Graphical structure of CRF

\section{Approach}

Over-Segmentation Algorithm. In order to combine with the recognition, segmentation algorithm we use is required with global features such as: pixels which belong to the different object shouldn't been segmented in the same region. In this section, we pre-partition each image into a set of contiguous regions using an over-segmentation algorithm, and segment the image by labeling these regions.

Toward probabilistic segmentation, we define a distributionover the labels of all regions in the image. Let $G=(V, E)$ be the graph to be segmented toregions, whereVis the set of pixels, Eis the set of edges, and each edge corresponds to a weight $u\left(v_{i}, v_{j}\right)$, which different from literature ${ }^{[7]}$, edge weight here defined as:

$$
u\left(v_{i}, v_{j}\right)=\left|g_{d}\left(v_{i}, v_{j}\right)\right|^{2}
$$

Where, $g_{d}\left(v_{i}, v_{j}\right)$ is the gray value difference of $v_{i}$ and $v_{j}$. The goal of segmentation is to find an region setR $=\left(B_{1}, \cdots, B_{k}\right)$, make $\cup B_{i} \in$ Vand wheni $\neq j, B_{i} \cap B_{j}=\emptyset$.

The discrepancy functionof single regionalis defined as 


$$
\operatorname{Int}(B)=\max _{\left(v_{i}, v_{j}\right) \in E, v_{i}, v_{j} \in B} u\left(v_{i}, v_{j}\right)
$$

The discrepancy functionof two regions is defined as

$$
\operatorname{TInt}\left(B_{i}, B_{j}\right)=\min \left(\operatorname{Int}\left(B_{i}\right)+\delta / M_{B_{i}}, \operatorname{Int}\left(B_{j}\right)+\delta / M_{B_{j}}\right)
$$

Where, $M_{B_{i}}, M_{B_{j}}$ is the pixel numbers of region $B_{i}, B_{j}$, when $B_{i=} B_{j=1}, \operatorname{Int}(B)=0$.Parameter $\delta$ is a constant,the $\delta$ is larger, the region we get is bigger,here $\delta=200$ 。

The Algorithm Process. Input: $\mathrm{G}=(\mathrm{V}, \mathrm{E})$, which include npixels, medges; Output: segment pixel set Vto region set $R=\left(B_{1}, \cdots, B_{k}\right)$.

Step1.Ascending sort $\mathrm{E}$ to $\left(\mathrm{o}_{1}, \cdots, \mathrm{o}_{\mathrm{m}}\right)$; Let initial value $\mathrm{R}_{0}$ be: each pixel ${ }^{v_{i}}$ to be a region; Forq $=1, \cdots \mathrm{m}$, repeat thestep2.

Step2.Use the follow method fromB ${ }^{\mathrm{q}-1} \operatorname{constructB}^{\mathrm{q}}$ : Letv $_{\mathrm{i}}, \mathrm{v}_{\mathrm{j}}$ to be the two pixels the edge connected, that is $o_{q}\left(v_{i}, v_{j}\right)$. If $v_{i} \in B_{i}^{q-1}, v_{j} \in B_{j}^{q-1}$ and $B_{i}^{q-1}, B_{i}^{q-1} \in R^{q-1}$ 。 When $B_{i}^{q-1} \neq$ $B_{i}^{q-1}$ andu $\left(o_{q}\right) \leq \operatorname{TInt}\left(B_{i}, B_{j}\right), \operatorname{merge} B_{i}^{q-1}, B_{i}^{q-1} \operatorname{toR}^{\mathrm{q}}$, else let $R^{q}=R^{q-1}$ 。

Step3.If region $R_{\mathrm{i}}$ is less than the minimum allowable area $\mathrm{R}_{\min }$, merge $B_{\mathrm{i}}$ and $\mathrm{B}_{\mathrm{j}}$ together,until all the regions are greater than $\mathrm{R}_{\mathrm{min}}$.Finally, $B_{j}^{\prime}=\min _{R_{j}} \operatorname{TInt}\left(B_{i}, B_{j}\right) \mathrm{andB}_{\mathrm{i}}$ andB $_{\mathrm{j}}$ are neighbored。

In this paper, we select lung CT image for processing, results of over-segmentation are shown in Figure 2.

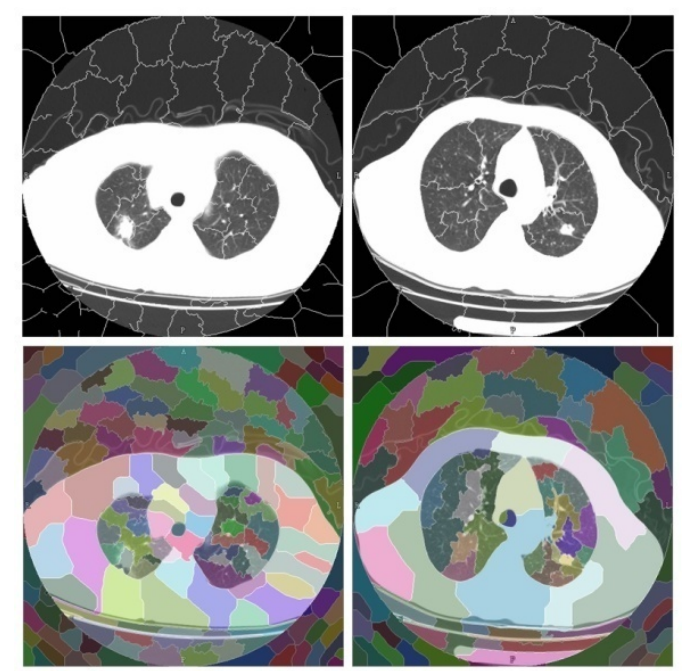

Fig.2Results of over-segmentation for lung CT

Region Based CRF Model. When creating graphical models, nodes are constituted by the region, edgesareundirected connections between nodes. Image over-segmentation algorithm is used first to segment image into relatively homogeneous regions, each region corresponds to a node in the CRF model. If two regions are adjacent in the over-segmentation, then joins an edge between their corresponding nodes in the CRF model. Thus the graphical model we constructed is irregular, different nodes may correspond to different numbers of adjacent nodes, while the graph model whose nodes are constituted by pixels is regular. Although pixel-based graphical model is relatively simple, but with the increase in image size, the number of nodes in the model increases, the computational complexity also increases. The region-based graph model greatly reduces the number of nodes, reducing the computational complexity, and nodes constructed by regions make the segmentation in homogeneousregion more consistently. Compared to the node constructed by pixels, its size is greatly reduced, which played a crucial role for reducing the complexity of parameter estimation and derivation.For a certain region $S_{\mathrm{i}}$ of image $\mathrm{X}$,the conditional probabilitybelonging to $\mathrm{C}_{\mathrm{i}}$ is defined as 


$$
P\left(C_{i}, S_{i} \mid X ; \theta\right) \propto \exp \left(\frac{\mathbf{1}}{\mathbf{s}_{\mathbf{i}}} \sum_{\mathbf{i} \in \mathbf{S}_{\mathbf{i}}} \ln \mathbf{p}\left(\mathbf{C}_{\mathbf{i}} \mid \mathbf{X} ; \boldsymbol{\theta}\right)\right)
$$

Where, the conditional probability $p\left(C_{i} \mid X ; \theta\right)$ that represents pixel i belongs to class $C_{i}$ and parameter $\theta$ has been obtained in our previous work [8]. We can get the class that region $S_{i}$ belongs to by maximizing the above conditional probability. Assign the same class to each pixel of region $S_{\mathrm{i}}$,we can achieve the segmentation and classificationbasedpixel.

\section{Experimental Results}

We selectlung CTimagefor segmentation, results are shown in Figure 3.We cansee that lesion area have been recognized from thesurrounding successfully, the overall accuracy we get is up to 84.5\%.As shown in Table 1, when the number of training samples is 134, the average running time of pixel-based CRF model for lung CT medical image labeling is 3.769s, and that of region-based CRF modelis 2.557s.Experimental results show that, the region-based CRF model get the desiredclassification results, it greatly reduces the run time and improve efficiency simultaneously.
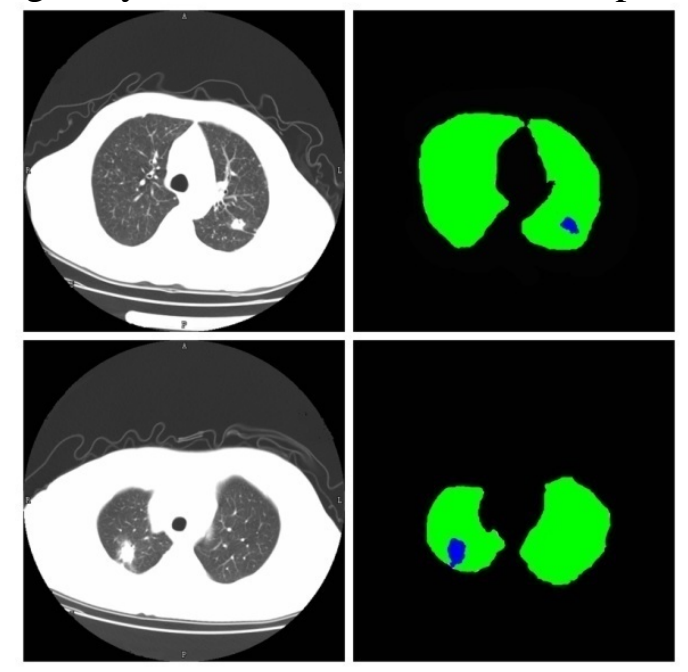

Fig.3Region based CRF for medical image labeling

Table 1Thecomparison of region-based CRF model and pixel-based CRF model

\begin{tabular}{ccc}
\hline methods(134 training samples) & Time PER & Accuracy \\
\hline region-based CRF model & $2.557 \mathrm{~s}$ & $82.97 \%$ \\
pixel-based CRF model & $3.769 \mathrm{~s}$ & $81.53 \%$ \\
\hline
\end{tabular}

\section{Summary}

This paper proposed a region-based CRF model for medical image labeling. Firstly, we use Image over-segmentation algorithm to segment image into small regions, and then establishthe CRF model with regional nodes. With respect to the pixel-based approach, region-based CRF model greatly reduces the number of nodes in the graph model, reducing the computational complexity, and the region is better to retain the internal structure information of the image. The experimental results show that the region-based CRF model getsthe desired medical image labeling results, and greatly reduces the run time and improves efficiency simultaneously.

\section{Acknowledgments}

Funded by grants from science and technology development plan project of Jilin province--The Youth Foundation (201201112) and scientific research of Jilin provincial education department (2014520) 


\section{References}

[1]Lafferty J, McCallum A, Pereira F C N. Conditional random fields: Probabilistic models for segmenting and labeling sequence data[J]. 2001.

[2]Wang Y, Loe K F, Wu J K. A dynamic conditional random field model for foreground and shadow segmentation[J]. Pattern Analysis and Machine Intelligence, IEEE Transactions on, 2006, 28(2): 279-289.

[3]He X, Zemel R S, Carreira-Perpindn M A. Multiscale conditional random fields for image labeling[C]//Computer vision and pattern recognition, 2004. CVPR 2004.Proceedings of the 2004 IEEE computer society conference on. IEEE, 2004, 2: II-695-II-702 Vol. 2.

[4]Zhou L, Qiao Y, Yang J, et al. Learning geodesic CRF model for image segmentation[C]//Image Processing (ICIP), 2012 19th IEEE International Conference on. IEEE, 2012: 1565-1568.

[5]Orlando J I, Blaschko M. Learning fully-connected CRFs for blood vessel segmentation in retinal images[M]//Medical Image Computing and Computer-Assisted Intervention-MICCAI 2014. Springer International Publishing, 2014: 634-641.

[6]Hammersley, J., \& Clifford, P. (1971). Markov fields on finite graphs and lattices. Unpublished manuscript.

[7]Felzenszwalb P F, Huttenlocher D P. Efficient graph-based image segmentation[J]. International Journal of Computer Vision, 2004, 59(2): 167-181.

[8]Yan Y, Huang W B, Wang Y J, et al. Image Labeling Model Based on Conditional Random Fields[J]. Advanced Materials Research, 2013, 756: 3869-3873. 